\title{
Preliminary evaluation of "Clearview Chlamydia" for the rapid detection of chlamydial antigen in cervical secretions
}

\author{
H Young, A Moyes, H Lough, I W Smith, J G McKenna, ` C Thompson`
}

\begin{abstract}
Clearview Chlamydia (Unipath) is a rapid monoclonal antibody based latex immunodiffusion test for detecting chlamydial antigen in endocervical specimens. The assay does not require specialised equipment or extensive training and takes less than 30 minutes from sample to results. The clinical performance of Clearview Chlamydia was evaluated with 478 paired endocervical swabs from patients attending a genitourinary medicine clinic. In the first part of the study, 221 non-randomised specimens were tested by cell culture (1st swab) and Clearview (2nd swab) whereas in the second part of the study 257 randomised swabs were examined by Clearview, cell culture and immunofluorescence. Theoverall prevalence of chlamydial infection was $8 \cdot 8 \%$ and the sensitivity, specificity, positive and negative predictive values for Clearview were $85.7 \%$, $99 \cdot 1 \%, 90 \%$ and $98.6 \%$. The test requires further evaluation to establish its role in the management and control of chlamydial infection.
\end{abstract}

\section{Introduction}

Infection with Chlamydia trachomatis is probably the most common sexually transmitted disease in the western world. ${ }^{1}$ In the case of men, presentation with a non-gonococcal urethritis (NGU) with urethral discharge will lead to the prescription of an appropriate effective antibiotic, whereas diagnosis in women on the grounds of clinical signs is quite unreliable and appropriate therapy will often not be given. Whilst the majority of infections in women may be asymptomatic, infection with $C$ trachomatis is capable of inducing a plasma cell endometritis, salpingitis and peritonitis with associated

Departments of Medical Microbiology and

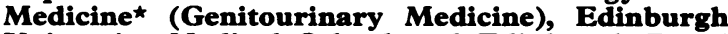
University Medical School and Edinburgh Royal Infirmary, Edinburgh, UK

H Young, A Moyes, H Lough, I W Smith, J G McKenna, * C Thompson* perihepatitis (Curtis Fitz-Hugh syndrome) and periappendicitis. ${ }^{2}$ Resolution of upper genital tract infection may result in chronic pelvic pain, tubal infertility or ectopic pregnancy.

The wide clinical spectrum of $C$ trachomatis infection combined with the lack of simple and rapid diagnostic methods is a major limitation in the control of chlamydial disease. ${ }^{3}$ Current diagnostic protocols include tissue culture and antigen detection by enzyme immunoassay and immunofluorescence (IF) staining which have time scales of days, 3-4 hours and less than an hour respectively. Although IF staining is rapid it requires highly skilled personnel, expensive equipment and can only be applied to relatively small numbers of specimens.

A simple, rapid and reliable test for chlamydial antigen that could be performed at the time of the patient's visit would be of enormous value in the management and control of infection. Clearview Chlamydia (Unipath) is a new simple and rapid (less than 30 minutes from sample to result) test that does not require specialised equipment or extensive training. Our aim was to evaluate the performance of this new test in female patients attending a genitourinary medicine clinic.

\section{Materials and methods}

\section{Patients}

A total of 478 new and returned-new female patients attending the Genitourinary Medicine Unit, Edinburgh Royal Infirmary were included in the study. In the first part of the study involving 221 patients a comparison was made between Clearview Chlamydia (antigen detection) and cell culture. The cervical swab for culture was always taken before the swab for antigen detection. The second part of the study compared the performance of Clearview Chlamydia, cell culture and IF in 257 patients. In this case the order of taking swabs was randomised: one swab was used for culture and the other used to prepare a smear for IF before extracting antigen for the Clearview test.

A retrospective analysis of the case notes was made to determine if the women were known contacts of 
men with non-gonococcal urethritis (NGU) and if they had received antibiotics within the preceding 10-14 days.

\section{Cell culture}

Swabs to be cultured for chlamydiae were expressed in sucrose-phosphate transport medium (2SP) which was then placed in a freezer at $-70^{\circ} \mathrm{C}$. Specimens were thawed and vortexed prior to culture in cycloheximide treated McCoy cells. ${ }^{5}$ Incubation of the infected cells was continued for three days at $35^{\circ} \mathrm{C}$. Cells were then stained with iodine and C. trachomatis was considered to be present if characteristic intracytoplasmic inclusions were seen. ${ }^{6}$

\section{Immunofluorescence (IF)}

Smears for IF were allowed to air dry and fixed in methanol. The Syva MicroTrak test was performed according to the manufacturer's instructions.

\section{Clearview Chlamydia}

Immediately after the smear had been prepared the swab was replaced in its sleeve (no transport medium) and transported to the laboratory within three hours. If the Clearview test was not performed the same day, swabs were stored at $4^{\circ} \mathrm{C}$ and tested within three days according to the manufacturer's instructions. The swab was placed in a small flexible plastic extraction tube containing $0.6 \mathrm{ml}$ extraction buffer and agitated. The extraction tube containing the swab was then placed in a heating block at $80^{\circ} \mathrm{C}$ for ten minutes. On removing the extraction tube from the heating block the swab was rotated in the buffer and removed from the tube: liquid was thoroughly removed from the swab by pinching the rim of the extraction tube between thumb and forefinger and squeezing the swab as it was removed from the tube. After cooling for five minutes at room temperature a drop of antibody coated latex suspension (blue latex particles coated with genus specific monoclonal antibody against chlamydial lipopolysaccharide) was added and mixed with the extract. The extraction tube was then capped with an integral cap which has an in-built filter and acts as a dropper for applying sample to the test device. Five drops of this mixture were then added to an absorbent pad in the sample window of a small $(8.5 \mathrm{~cm} \times 3.0 \mathrm{~cm})$ immunochromatographic device. The mixture migrates from the sample window along a strip to the "Result Window": the formation of a blue line in the result window (within 5 minutes) indicates the presence of chlamydial antigen in the extract. The line is formed due to the binding of chlamydial antigen to the blue latex and its immobilisation by a zone of antibody located beneath the result window. If no antigen is present the result window remains clear. Formation of a blue line in a "Control Window" shows that the test has been performed correctly. The control line results from binding of some of the antibody sensitised latex by an immobilised zone of antibodies to mouse immunoglobulin. Test performance was validated by appropriate positive and negative controls.

\section{Results}

In the first part of the study a total of 20 patients gave a positive result by culture and/or Clearview. The correlation between the two methods is shown in table 1.

Based on culture the sensitivity of Clearview was $88.9 \%(16 / 18)$ and the specificity was $99 \%(201 / 203)$. Neither of the patients with an unconfirmed Clearview result was a known contact of NGU although one of the women had gonorrhoea.

The result pattern for the 257 patients examined by all three tests in the second part of the study is shown in table 2.

All three tests were negative in 230 patients and postive in 18 giving an overall correlation of $96.5 \%$ (248/257).The nine discrepancies comprised two patients with a positive Clearview test confirmed by IF, two with a positive IF test confirmed by culture, two with a positive culture only, two with an unconfirmed Clearview test, and one with an unconfirmed IF test. This last patient had received antibiotic at a family planning clinic prior to the chlamydial culture being taken and was excluded from further analysis. Of the 24 positive chlamydia cases only $15(62.5 \%)$ were known contacts of men with NGU. Eight of the nine women not known to be contacts of men with NGU were Clearview positive. Of the 232 chlamydia negative patients $24(10 \cdot 3 \%)$ were known contacts of men with NGU.

The overall correlation between Clearview and

Table 1 Correlation between Clearview and culture for 221 female patients

\begin{tabular}{lccc}
\hline & Clearview Pos & Clearview Neg & Total \\
\hline Culture positive & 16 & 2 & 18 \\
Culture negative & 2 & 201 & 203 \\
Total & 18 & 203 & 221 \\
\hline
\end{tabular}

Table 2 Test result pattern for 257 female patients

\begin{tabular}{lllc}
\hline Clearview & IF & Culture & Number of specimens \\
\hline Neg & Neg & Neg & 230 \\
Pos & Pos & Pos & 18 \\
Pos & Pos & Neg & 2 \\
Neg & Pos & Pos & 2 \\
Neg & Neg & Pos & 2 \\
Neg & Pos & Neg & 1 \\
Pos & Neg & Neg & 2 \\
\hline
\end{tabular}

IF $=$ Immunofluorescence

Correlation for three tests: $96.5 \%(248 / 257)$ 
Table 3 Overall correlation between Clearview and chlamydial infection detected by culture and/or IF for 477 female patients

\begin{tabular}{lccc}
\hline & Clearview Pos & Clearview Neg & Total \\
\hline Culture/IF Positive & 36 & 6 & 42 \\
Culture/IF Negative & 4 & 431 & 435 \\
Total & 40 & 437 & 477 \\
\hline
\end{tabular}

detection of chlamydia by IF and/or cell culture is shown in table 3.

The overall prevalence of chlamydial infection was $8.8 \%(42 / 477)$ and the sensitivity and specificity of Clearview were $85 \cdot 7 \%(36 / 42)$ and $99 \cdot 1 \%(431 / 435)$ respectively. The corresponding positive and negative predictive values were $90 \%(36 / 40)$ and $98 \cdot 6 \%(431 / 437)$.

\section{Discussion}

Non-cultural methods for the diagnosis of chlamydial infection are widely used but none is wholly reliable and discrepancies may occur. ${ }^{7}$ It is therefore necessary to ensure that new tests are evaluated in such a way as to determine as accurately as possible whether the results agree with those of the best techniques available. ${ }^{8}$ Although cell culture is often taken as the gold standard for detection of $C$. trachomatis infection it is now generally accepted that the sensitivity of culture is less than $100 \% .^{249}$ Because the sensitivity of culture may be less than $100 \%$ and as non-cultural methods detect non-viable organisms a third test (IF) was used to help resolve any discrepancies.

The sensitivity and specificity of Clearview as determined against the combination of cell culture and IF were $85 \cdot 7 \%(36 / 42)$ and $99 \cdot 1 \%(431 / 435)$ with a corresponding positive predictive value of $90 \%$ and a negative predictive value of $98.6 \%$. The overall agreement between all three tests was $96 \cdot 5 \%$ which is almost identical to the overall agreement of 97\% between the Pharmacia EIA, IF and cell culture. $^{8}$ The sensitivity, specificity, positive and negative predictive values for the Pharmacia EIA in detecting endocervical chlamydial infection were $86 \%, 97 \cdot 2 \%, 87 \cdot 8 \%$ and $96 \cdot 8 \%$ respectively. ${ }^{8}$

Chlamydiazyme (Abbott Diagnostics) is the most widely evaluated EIA and reported sensitivities in detecting endocervical chlamydial infection generally range from $70-100 \%$ with most studies in the 85-95\% range. ${ }^{4}$ Taylor-Robinson $e t a^{10}$ reported a poorer performance for Chlamydiazyme with a sensitivity of $67 \%$ and a specificity of $89 \%$ for endocervical specimens. False positive Chlamydiazyme tests may be due to cross-reaction with other organisms: various bacteria including Acinetobacter spp., Klebsiella spp., Streptococcus spp., and Gardnerella spp. at concentrations of $>10^{5} / 1$ react in the Chlamydiazyme assay to yield false positive results. ${ }^{11}$ Similar performance parameters have been reported between Chlamydiazyme and other EIAs such as the amplified EIA, IDEIA (Novo Bio Labs, formerly Celltech Diagnostics).$^{12}$ Mahoney et al ${ }^{13}$ however, found the sensitivity of IDEIA (96.3\%) was higher than that of Chlamydiazyme $(85 \cdot 2 \%)$. The IDEIA III version of the assay which used Fab fragments rather than whole monoclonal immunoglobulin $G$ as the capture antibody yielded sensitivity, specificity, positive and negative predictive values of $93.8 \%, 99 \%, 92.9 \%$ and $99 \cdot 1 \%$ respectively. ${ }^{14}$

Processing of samples by the above non-cultural assays takes several hours and although they are ideally suited to batch testing in the laboratory they cannot be used as an "on-the-spot" test when the patient is at the clinic. The new rapid tests which are available yield similar results to the above EIAs but can be performed at the time of the patient's visit. TestPack Chlamydia (Abbott Laboratories) is a direct EIA for the detection of chlamydial antigen in endocervical specimens. ${ }^{15}$ The assay requires no specialised equipment and yields results in less than 30 minutes. When the assay was evaluated against cell culture and/or chlamydial antigen positive (confirmed Chlamydiazyme or IF staining) in a population with a prevalence of chlamydial infection of $13.8 \%$ the assay yielded a sensitivity, specificity, positive and negative predictive values of $76.5 \%$, $99.5 \%, 96.2 \%$ and $96.5 \%$ respectively..$^{15}$ In our study Clearview Chlamydia gave a slightly higher sensitivity $(85.7 \%)$ in a population with a lower prevalence $(8.8 \%)$ of chlamydial infection. The various factors that must be taken into account when selecting tests for chlamydial infection are discussed in detail by Barnes. ${ }^{4}$ The development of these new tests, however, means that the advantages of rapid antigen detection thus enabling the patient to receive immediate effective treatment require greater consideration. The use of these immediate tests must be deliberated against the setting where epidemiological treatment is widely practised. ${ }^{1}$ Within Genitourinary medicine clinics epidemiological treatment is usually given to women who are known contacts of men with NGU. In this context it is interesting that $40 \%(8 / 20)$ of the Clearview positive patients were not known contacts of men with NGU and would not have been treated on epidemiological grounds. In contrast, $10.3 \%(24 / 232)$ of the chlamydia negative patients were contacts of men with NGU and would have received epidemiological treatment. Despite epidemiological treatment the knowledge of whether a patient is chlamydia positive or negative may be useful, particularly when there is a risk to other contacts.

Because culture following storage at $-70^{\circ} \mathrm{C}$ may result in a slightly decreased sensitivity it is possible 
that we have over-estimated the sensitivity of Clearview. Nevertheless the test obviously merits further evaluation against stricter diagnostic methodology. Assuming that our favourable results are confirmed the test could be of value in several situations where rapid detection of chlamydial infection is important. For example, there is a need to screen women before vaginal termination of pregnancy. ${ }^{1617}$ Greater access to chlamydial diagnosis would also be helpful in managing women with pelvic inflammatory disease who present to gynaecology departments, ${ }^{18}$ women who attend family planning clinics ${ }^{19}$ and who attend inner city general practices. $^{20-22}$

We thank our clinical and laboratory colleagues in Genitourinary Medicine and Medical Microbiology, particularly Alistair McCulloch, for their help and cooperation with this study.

Address for correspondence: Dr H Young, Department of Medical Microbiology, Edinburgh University Medical School, Teviot Place, Edinburgh EH8 9AG, UK.

1 Royal College of Physicians Committee on Genitourinary Medicine. Chlamydial diagnostic services in the United Kingdom and Eire: current facilities and perceived needs. Genitourin Med 1987;63:371-4.

2 Treharne JD, Ballard RC. The expanding spectrum of the Chlamydia - a microbiological and clinical appraisal. Rev Med Microbiol 1990;1:10-18.

3 Kuo C-C, Tam MR. Immunological diagnosis of Chlamydia trachomatis infection. In: Young H, McMillan E, eds. Immunological Diagnosis of Sexually Transmitted Diseases. New York: Marcel Dekker. 1988;192-211.

4 Barnes RC. Laboratory diagnosis of human chlamydial infections. Clin Microbiol Rev 1989;2:119-36.

5 Thomas BJ, Evans RT, Hutchinson GR, Taylor-Robinson D. Early detection of Chlamydia inclusions combining the use of cycloheximide treated McCoy cells and immunofluorescence staining. J Clin Microbiol 1977;6:285-92.

6 Evans RT, Woodland RM. Detection of chlamydiae by isolation and direct examination. Br Med Bull 1983;39:181-6.
7 Ridgway GL. The laboratory diagnosis of chlamydial infection. In: Oriel JD, Ridgway GL, Schachter J, Taylor-Robinson D, Ward $\mathbf{M}$, eds. Chlamydial Infection. London: Cambridge University Press. 1986;539-49.

8 Mumtaz G, Ridgway GL, Nayagam A, Oriel JD. Enzyme immunoassay compared with cell culture and immunofluorescence for detecting genital chlamydia. J Clin Pathol 1989; 42:658-60.

9 Jones RB, van der Pol B, Katz BP. Effect of differences in specimen processing and passage technique on recovery of Chlamydia trachomatis. J Clin Microbiol 1989;27:894-8.

10 Taylor-Robinson D, Thomas BJ, Osborn MF. Evaluation of enzyme-immunoassay (Chlamydiazyme) for detecting C. trachomatis in genital tract specimens. J Clin Pathol 1987;40: 194-9.

11 Moncada J, Schachter J, Bolan G, et al. Confirmatory assay increases specificity of the Chlamydiazyme test for Chlamydia trachomatis infection of the cervix. J Clin Microbiol 1990; 28:1770-3.

12 Mohanty KC, O'Neill JJ, Hambling MH. Comparison of enzyme immunoassays and cell culture for detecting Chlamydia trachomatis. Genitourin Med 1986;62:175-6.

13 Mahoney J, Castriciano S, Sellors J, et al. Diagnosis of Chlamydia trachomatis genital infections by cell culture and two enzyme immunoassays detecting different chlamydial antigens. J Clin Microbiol 1989;27:1934-8.

14 Lebar WD, Schubiner H, Jemal C, Herschman BR. Comparison of IDEIA III and cell culture for the detection of Chlamydia trachomatis in endocervical specimens. J Clin Microbiol 1990; 28:1447-8.

15 Coleman P, Varitek V, Mushahwar IK, et al. TestPack Chlamydia, a new rapid assay for the direct detection of $C$. trachomatis. J Clin Microbiol 1989;27:2811-4.

16 Osser S, Persson K. Post-abortal pelvic infection associated with Chlamydia trachomatis and the influence of humoral immunity. Am J Obstet Gynecol 1984;150:699-703.

17 Southgate LJ, Treharne JD, Williams R. Detection, treatment and follow-up of women with Chlamydia trachomatis infection seeking abortion in inner city general practices. BMJ 1989; 299:1136-7.

18 Scott GR, Thompson C, Smith IW, Young H. Infection with Chlamydia trachomatis and Neisseria gonorrhoeae in women with lower abdominal pain admitted to a gynaecology unit. $\mathrm{Br}$ J Obstet Gynaecol 1989;96:473-7.

19 Webb AMC, Chandiok S. Women with Chlamydia trachomatis infection. $B M J$ 1989;299:1527.

20 Longhurst HJ, Flower F, Thomas BJ, et al. A simple method for the detection of Chlamydia trachomatis infections in general practice. J R Coll Gen Pract 1987;37:255-6.

21 Harrison N, Smith IW, Young H. Chlamydia trachomatis infection in sexually active women with no known sexual contacts with urethritis. Scot Med J 1989;34:464-6.

22 Southgate LJ, Treharne JD, Forsey T. Chlamydia trachomatis and Neisseria gonorrhoeae infections in women attending inner city general practices. BMJ 1983;287:879-81.

Accepted for publication 21 December 1990 